\title{
Los niveles de mediadores prorresolutivos no corresponden con los de mediadores inflamatorios en enfermedades isquémicas coronarias
}

\author{
Rashidi Springall-del Villar, ${ }^{1}$ Jorge Arturo Ortega-Hernández, ${ }^{1,2}$, Luis Fernando Pérez,,3 \\ Fausto Sánchez-Muñoz, ${ }^{1}$ Julio César Arana Martínez,, ${ }^{1,3}$ Héctor González-Pacheco 4 y Rafael Bojalil1,3 \\ ${ }^{1}$ Departmento de Inmunología, Instituto Nacional de Cardiología Ignacio Chávez; ${ }^{2}$ Facultad de Medicina, Universidad Nacional Autónoma de México; \\ ${ }^{3}$ Departmento de Atención a la Salud, Universidad Autónoma Metropolitana Xochimilco; ${ }^{4}$ Unidad de Cuidados Coronarios, Instituto Nacional de \\ Cardiología Ignacio Chávez. Ciudad de México, México
}

\section{Resumen}

Antecedentes: La inflamación puede agruparse en tres fases: proinflamatoria, antiinflamatoria y de resolución. Esta última, principalmente atribuida a mediadores lipídicos, es la de más reciente descripción y se ha estudiado poco en las enfermedades isquémicas coronarias. Objetivos: Evaluar 1) si los síndromes coronarios agudos (SICA) manifiestan niveles circulantes distintos de mediadores de resolución comparados con la angina estable (AE); 2) si sus concentraciones se relacionan con las de mediadores proinflamatorios y antiinflamatorios; $y$ 3) si dichas concentraciones se asocian con la gravedad de la enfermedad y el daño producido. Método: Se midieron LTB4, RVD1, LXA4, ET-1, MMP-2, MMP-9, TIMP-1, IL-1及, IL-6, IL-8 e IL-10 en suero. Se establecieron la puntuación GRACE como parámetro de gravedad y la fracción de eyección del ventrículo izquierdo (FEVI) como parámetro de daño. Resultados: Se incluyeron 30 pacientes con AE, 37 con SICA sin elevación del segmento ST (SICA-SEST), 38 con Infarto agudo del miocardio con elevación del segmetno ST(IAM-CEST) y 10 con dolor torácico no cardiogénico. Los pacientes con enfermedad coronaria mostraron niveles elevados de citocinas inflamatorias y bajos de mediadores de resolución. Conclusiones: La escasa respuesta de resolución aun en pacientes con enfermedad coronaria aguda sugiere una incapacidad para reparar daños. Probar esta hipótesis tendría el potencial de sugerir nuevas terapias para el manejo de la inflamación cardiovascular crónica.

PALABRAS CLAVE: Inflamación. Mediadores de resolución. Síndromes coronarios.

\section{Abstract}

Background: Inflammation can be grouped into three phases: proinflammatory, anti-inflammatory, and resolution. The latter, mainly attributed to lipid mediators, is the most recently described, and has been studied little in coronary ischemic diseases. Objective: To evaluate 1) if acute coronary syndromes (ACS) manifest different circulating levels of resolution mediators compared with stable angina (SA); 2) if their concentrations are related to those of pro and anti-inflammatory mediators; and 3) if such concentrations are associated with the severity of the disease and the damage produced. Method: LTB4, RvD1, LXA4, ET-1, MMP-2, MMP-9, TIMP-1, IL-1 $\beta$, IL-6, IL-8 and IL-10 were measured in serum. The GRACE score was established as parameter of gravity, and LVEF as a damage parameter. Results: Thirty patients with SA, 37 with NEST-ACS, 38 with STEMI, and 10 individuals with non-cardiogenic chest pain were included. Patients with coronary artery disease showed elevated levels of inflammatory cytokines and low levels of resolution mediators. Conclusions: The low resolution response even in patients with acute coronary disease suggests an inability to repair damage. Testing this hypothesis would have the potential to suggest new therapies for the management of chronic cardiovascular inflammation.

KEY WORDS: Inflammation. Resolution mediators. Coronary syndromes.

Correspondencia:

Rafael Bojalil

E-mail: rafaelbojalil@gmail.com
Fecha de recepción: 16-08-2018

Fecha de aceptación: 20-08-2018

DOI: 10.24875/GMM.18004572
Gaceta Med Mex. 2018;154(Suppl 2): S9-S14

Disponible en PubMed www.gacetamedicademexico.com 


\section{Introducción}

Durante casi todos los procesos que implican un daño tisular o una disfunción, de origen endógeno 0 exógeno, se produce por parte del organismo una respuesta inflamatoria en la que pueden distinguirse tres fases: proinflamatoria, antiinflamatoria y de resolución. La fase de resolución tiene como función restaurar la homeostasis tisular y favorecer la reparación de los tejidos. ${ }^{1,2}$ Si la inflamación no es resuelta completamente puede generarse un estado de inflamación persistente, que tiene un papel fundamental en el desarrollo de enfermedades crónicas. ${ }^{2,3}$ Específicamente, en la aterosclerosis y las enfermedades cardiovasculares, muy vinculadas entre sí, existe mucha evidencia que las relaciona con una inflamación crónica no resuelta. ${ }^{4,5}$ Las enfermedades cardiovasculares incluyen la angina estable (AE) y el conjunto de los síndromes coronarios agudos (SICA). Los SICA abarcan la angina inestable y el infarto agudo del miocardio (IAM) sin elevación del segmento ST (SEST), que suelen agruparse en el denominado SICA-SEST; incluyen también el IAM con elevación del segmento ST (IAM-CEST). ${ }^{6}$ Los fenómenos inflamatorios asociados a las enfermedades isquémicas coronarias han sido objeto de una gran cantidad de estudios, ${ }^{7-10} \sin$ embargo, los procesos que resuelven la inflamación se encuentran poco estudiados, y esto se ha hecho fundamentalmente en modelos animales. ${ }^{11}$

La resolución de un proceso inflamatorio no depende solo de la reducción de la concentración de mediadores proinflamatorios, ya sea por disminución de su producción o por la presencia de mediadores antagónicos. La resolución activa de la inflamación depende de un conjunto de mediadores especializados que inhiben la diapédesis y estimulan la salida del tejido de células inflamatorias, además de favorecer la fagocitosis de células en apoptosis (eferocitosis). Estos mediadores también favorecen la eliminación de fragmentos celulares y la reparación de tejidos. ${ }^{12}$ Las moléculas de resolución incluyen lípidos derivados del ácido araquidónico (lipoxinas) y de los ácidos eicosapentaenoico y docosahexaenoico: resolvinas, protectinas y maresinas. En conjunto se conocen como mediadores lipídicos resolutivos especializados. ${ }^{12}$ En diversas enfermedades crónicas, incluyendo la $A E$, se ha demostrado la presencia de niveles circulantes bajos de mediadores lipídicos prorresolutivos. ${ }^{3}$ Por el contrario, en una infección aguda se elevan los niveles de dichos mediadores, de cuya producción depende la resolución. ${ }^{13}$ De ahí la importancia de indagar si después de un evento coronario agudo se elevan los niveles de resolvinas y lipoxinas.

Para ello diseñamos un estudio con el fin de evaluar: 1) si los pacientes con SICA manifiestan niveles circulantes distintos de mediadores de resolución comparados con los pacientes con AE; 2) si sus concentraciones se relacionan con las de mediadores proinflamatorios y antiinflamatorios; y 3) si dichas concentraciones se asocian con la gravedad de la enfermedad.

\section{Método}

\section{Sujetos de estudio}

En este estudio descriptivo y transversal se incluyeron pacientes admitidos a la unidad coronaria y provenientes de la consulta externa de cardiología, del Instituto Nacional de Cardiología Ignacio Chávez. El estudio fue aprobado por los comités de investigación y de ética. Todos los pacientes o sus representantes legales firmaron un consentimiento informado.

Se formaron cuatro grupos: 1) individuos que acudieron a la unidad coronaria por dolor torácico y en quienes se descartó un origen cardiaco del dolor; 2) pacientes con diagnóstico de $\mathrm{AE}$ atendidos en la consulta externa, reclutados antes del inicio de cualquier tratamiento; 3) pacientes con diagnóstico de angina inestable o de IAM sin elevación del segmento ST, agrupados como SICA-SEST; 4) pacientes con diagnóstico de IAM-CEST. Todos los diagnósticos se hicieron de acuerdo con las guías norteamericanas actuales. ${ }^{6}$

Se obtuvieron datos antropométricos y demográficos de todos los individuos, incluyendo edad, sexo, índice de masa corporal, tratamiento actual e historia médica. Se excluyeron aquellos pacientes menores de 18 años, con enfermedades infecciosas, autoinmunitarias o neoplásicas, con insuficiencia renal grave (con filtración glomerular $<30 \mathrm{ml} / \mathrm{min}$ por $1.73 \mathrm{~m}^{2}$, estimada por la fórmula de Cockcroft-Gault) ${ }^{14} 0$ tratados con estatinas, aspirina, antiinflamatorios no esteroideos o esteroides. Se estableció la puntuación GRACE como parámetro de gravedad, ${ }^{15}$ y la fracción de eyección del ventrículo izquierdo (FEVI), medida por ecocardiografía, como parámetro de disfunción miocárdica. ${ }^{16}$

Este estudio se realizó de acuerdo con los principios de la Declaración de Helsinki y fue aprobado por la Comisión de Bioética del Instituto Nacional de Cardiología Ignacio Chávez. Todos los participantes firmaron una carta de consentimiento informado antes de la recolección de los datos. 
Tabla 1. Datos demográficos y clínicos de los sujetos de estudio

\begin{tabular}{|c|c|c|c|c|c|}
\hline & $\begin{array}{c}\text { Dolor torácico } \\
n=10\end{array}$ & $\begin{array}{c}\text { Angina estable } \\
n=30\end{array}$ & $\begin{array}{c}\text { SICA-SEST } \\
n=37\end{array}$ & $\begin{array}{c}\text { IAM-CEST } \\
n=38\end{array}$ & $\mathrm{p}$ \\
\hline Edad, años & $58(47-70)$ & $64(57-70)$ & $62(53-69)$ & $59(52-67)$ & 0.647 \\
\hline Hombres, n (\%) & $2(20)$ & $16(53.3)$ & $23(62.2)^{\star}$ & $32(84.2)^{\star,+, \neq}$ & 0.002 \\
\hline \multicolumn{6}{|l|}{ Factores de riesgo, n (\%) } \\
\hline Hipertensión & $6(60)$ & $20(66.7)$ & $21(56.8)$ & $22(57.9)$ & 0.923 \\
\hline Diabetes & $1(10)$ & $8(26.7)$ & $15(40.5)$ & $19(50)$ & 0.133 \\
\hline Dislipidemia & $2(20)$ & $7(23.3)$ & $13(35.1)$ & $11(29.7)$ & 0.087 \\
\hline IMC & $27(24.5-32.7)$ & $26.6(24.1-31.2)$ & $27.1(25.3-30.1)$ & $26(24.2-28.9)$ & 0.872 \\
\hline Tabaquismo & $3(30)$ & $14(46.7)$ & $21(56.7)$ & $26(72.2)^{*}$ & 0.047 \\
\hline \multicolumn{6}{|l|}{ Datos de laboratorio } \\
\hline Colesterol total, mg/dl & ND & $137.5(114.6-189.9)$ & $144.4(136.1-175.2)$ & $165.1(125.1-189.6)$ & 0.608 \\
\hline Colesterol LDL, mg/dl & ND & $87.4(61.0-117.3)$ & $91.5(78.4-111.3)$ & $108.6(79.9-133.0)$ & 0.171 \\
\hline Colesterol HDL, mg/dl & ND & $43.4(34.4-50.7)$ & $35.9(26.2-45.7)$ & 36.4 (32.5-42.1) & 0.158 \\
\hline Triglicéridos, mg/dl & ND & $105.5(93.3-157.3)$ & $132.3(99.2-197.1)$ & $128.4(100.5-184.2)$ & 0.596 \\
\hline PCR, mg/l & ND & $1.9(1.1-16.6)$ & $5.7(2.5-14.5)$ & $14.9(5.8-77.3)^{\dagger}$ & 0.008 \\
\hline
\end{tabular}

\section{Ensayos de mediadores en suero}

Al reclutamiento se obtuvieron $10 \mathrm{cc}$ de sangre, se centrifugaron a $600 \mathrm{~g}$ por 15 minutos a $7^{\circ} \mathrm{C}$ y las alícuotas de suero se almacenaron a $-76^{\circ} \mathrm{C}$ hasta su uso.

Las siguientes determinaciones se hicieron utilizando kits comerciales para Enzime-linked immunosorbent assay (ELISA): ET-1, LTB4, MMP-9, MMP-2 y TIMP-1 (R\&D Systems ${ }^{\mathrm{TM}}$ Inc., Minneapolis, MN, EE.UU.); RVD1 (Cayman Chemical Company, Ann Arbor, MI, EE.UU.); y LXA4 (Cloud-Clone Corp., Houston, TX, EE.UU.). Para IL-1 $\beta$, IL-6, IL-8 e IL-10 se usaron kits de inmunoensayos Multiplex (ProcartaPlex $^{\circledR}$ Bender, MedSystems GmbH, Viena, Austria) y se leyeron en un Luminex ${ }^{\circledR}$ MAGPIX (Luminex Corporation, Austin, Texas, EE.UU.) con software v4.2 (Lumine ${ }^{\circledR}{ }^{x}$ Ponent ${ }^{\circledR}$ for MAGPIX ${ }^{\circledR}$ ).

Los datos categóricos se describieron como frecuencias y porcentajes, y las diferencias se probaron por la prueba de ji al cuadrado. Para variables continuas se utilizaron medianas y rangos intercuartilares, y la prueba de Kruskal-Wallis (con corrección post hoc de Dunn-Bonferroni) se usó para comparar más de dos grupos, mientras que la comparación entre dos grupos se hizo con la prueba $U$ de Mann-Whitney. Las asociaciones se evaluaron utilizando la correlación de Spearman. Todos los análisis fueron a dos colas y se consideró significativo un valor de $p<0.05$. Los análisis se hicieron utilizando la versión 13.2.0 del software JMP (SAS Institute Inc.).

\section{Resultados}

\section{Población de estudio}

Se incluyeron en el estudio 115 participantes: 10 con dolor torácico de origen no cardiaco, 30 con AE, 37 con SICA-SEST y 38 con IAM-CEST. Las características clínicas principales se resumen en la Tabla 1. Como se puede observar, se encontraron diferencias significativas entre los grupos en sexo, tabaquismo y proteína $C$ reactiva (PCR). En las comparaciones pareadas, las diferencias significativas encontradas fueron todas relativas a pacientes con IAM-CEST: en sexo y tabaquismo se encontraron con los individuos con dolor torácico, y la PCR fue diferente con respecto a los pacientes con $\mathrm{AE}$.

\section{Concentraciones séricas de mediadores}

La Tabla 2 muestra el resumen de los hallazgos en los mediadores séricos. Encontramos diferencias estadísticas entre grupos para todos los mediadores estudiados, a excepción de LTB4 y TIMP-1. Al hacer el análisis por pares, los individuos con dolor torácico tuvieron niveles significativamente mayores de ambos mediadores de resolución (RvD1, LXA4) comparados con los tres grupos de pacientes con enfermedad coronaria, que no tuvieron diferencias entre sí. En sentido opuesto, los individuos con dolor torácico presentaron concentraciones séricas menores de MMP-2, MMP-9, IL-1 $1 \beta$, IL-6 e IL-8 que todos los grupos de pacientes. De manera 
Tabla 2. Valores de las determinaciones de mediadores en suero

\begin{tabular}{|c|c|c|c|c|c|}
\hline $\begin{array}{l}\text { Mediana, } \\
\text { RIQ (25-75) }\end{array}$ & $\begin{array}{l}\text { Dolor torácico } \\
\qquad n=10\end{array}$ & $\begin{array}{c}A E \\
n=30\end{array}$ & $\begin{array}{c}\text { SICA-SEST } \\
n=37\end{array}$ & $\begin{array}{c}\text { IAM-CEST } \\
n=38\end{array}$ & $\mathrm{p}$ \\
\hline LTB4, pg/ml & $1516(1088-1925)$ & $1698(1447-2104)$ & $1684(13234-2028)$ & 1397 (944-1939) & 0.4487 \\
\hline $\mathrm{RvD1}, \mathrm{ng} / \mathrm{ml}$ & $85.8(85.7-85.9)$ & $78.3(77.7-79.2)^{\star}$ & $79.2(78.4-79.8)^{\star}$ & $79.1(78.4-79.9)^{\star}$ & $<0.0001$ \\
\hline LXA4, pg/ml & $16.7(13.5-18.4)$ & $8(7-9)^{*}$ & $7.5(5.8-8.9)^{\star}$ & $8.5(7.2-9.1)^{*}$ & 0.001 \\
\hline $\mathrm{ET1}, \mathrm{pg} / \mathrm{ml}$ & $46.1(37.1-49.1)$ & $44(29-63)$ & $62.8(38.3-84.3)$ & $57.9(40.3-80.4)$ & 0.02 \\
\hline MMP-2, pg/ml & $1593(1430.7-1842.2)$ & $2486(2016-2892)^{*}$ & $2474.1(2010.3-2984.2)^{*}$ & $2427.7(2056.7-2984.2)^{*}$ & 0.0007 \\
\hline MMP-9, pg/ml & $1122(986.2-1354.7)$ & $4380(2806-5677)^{\star}$ & $4554(3312.0-5310.1)^{\star}$ & $4818.8(3410.2-5342.9)^{*}$ & $<0.0001$ \\
\hline TIMP-1, pg/ml & $488.2(461.3-561.8)$ & $515(389-604)$ & $563.0(422.7-784.4)$ & $548.9(411.4-647.1)$ & 0.547 \\
\hline $\mathrm{IL}-1 \beta, \mathrm{pg} / \mathrm{ml}$ & $0.5(0.5-0.5)$ & $59(0.5-114)$ & $114.0(0.5-242.9)^{\star}$ & $97.1(0.5-418.8)^{*}$ & 0.005 \\
\hline IL6, pg/ml & $227.3(17.3-753.4)$ & $941(548-1448)$ & $2254.5(647.3-6241.6)^{*}$ & $4190.3(1478.5-11700)^{*+}$ & $<0.0001$ \\
\hline IL-8, pg/ml & $368.9(244.8-518.6)$ & $688(460-1140)$ & $761.5(583.0-1289.5)^{\star}$ & $848.3(654.3-1586)^{*}$ & 0.013 \\
\hline IL10, pg/ml & $122.8(80.4-353.4)$ & $244(114-597)$ & $297.1(173.2-1121.5)$ & $861.9(273.0-1987.5)^{*+}$ & 0.004 \\
\hline
\end{tabular}

notable, en todos estos mediadores no hubo diferencias entre los grupos de pacientes, a excepción de la IL-6, para la que encontramos diferencias entre pacientes con IAM-CEST y aquellos con AE. La IL-10 tuvo un comportamiento similar en ese sentido y fue significativamente más alta en los pacientes con IAM-CEST que en aquellos con AE. La IL-10 también se encontró más elevada en los pacientes con IAM-CEST que en los individuos con dolor. En la comparación por pares no se encontraron diferencias en los niveles de ET-1.

\section{Correlaciones entre mediadores}

Todas las citocinas, tanto las inflamatorias (IL-1, IL-6, IL-8) como la antiinflamatoria (IL-10), correlacionaron directamente entre ellas, con $r$ de 0.44 a 0.72 y $p<0.0001$ para todas las correlaciones. De manera opuesta, todas las citocinas correlacionaron inversamente con los lípidos resolutivos; aquellas con RvD1 no alcanzaron significancia, y aquellas con LXA4 tuvieron $r$ de 0.24 a 0.29 y $p<0.001$ en todos los casos. También encontramos correlaciones inversas y significativas de MMP-2 con RvD1 ( $r=0.27$ y $p=0.003$ ), $y$ de MMP-9 con RvD1 y LXA4 $(r=0.45$ y 0.20 ; $p=0.008$ y 0.034 , respectivamente). Con respecto a las citocinas, MMP-2 correlacionó directamente con IL-6 $(r=0.30$ y $p=0.001)$ y MMP-9 con IL-1 e IL-6 ( $r=0.19$ y $0.21 ; p=0.04$ y 0.03 , respectivamente). Ambas metaloproteasas correlacionaron directamente entre sí, con $r=0.26$ y $p=0.006$. RvD1 y LXA4 también correlacionaron entre ellas de manera directa, con $r=0.20$ y $p=0.029$ ).

\section{Correlaciones entre la gravedad de la enfermedad y el daño miocárdico con los mediadores séricos}

Las puntuaciones GRACE correlacionaron positivamente con las concentraciones séricas de ET-1, IL-1, IL-6, IL-8 e IL-10 ( $r=0.303,0.311,0.448,0.304$ y $0.321 ; p=0.009$, $0.007,<0.0001,0.008$ y 0.005 , respectivamente).

La FEVI correlacionó negativamente con ET-1, IL-6, IL-8 e IL-10 ( $r=-0.351,-0.430,-0.534$ y -0.377 ; $p=0.036,0.009,0.0008$ y 0.024 , respectivamente).

\section{Discusión}

En este estudio transversal y observacional encontramos que las concentraciones séricas de RvD1 y LXA4, mediadores de resolución del proceso inflamatorio, son similares en todos los pacientes (con SICA, con o sin elevación del segmento ST, y con AE), quienes tienen concentraciones significativamente más bajas que las de los individuos con dolor torácico no cardiogénico. Es decir, contrario a lo esperado para un proceso agudo que logra resolverse, nuestros resultados indican que, a pesar de sufrir un daño agudo miocárdico, los pacientes con SICA no elevaron sus 
niveles de RvD1 y de LXA4 en circulación. En teoría, ello favorecería la persistencia de la infamación y de los trastornos en la reparación de los tejidos.

La baja concentración de mediadores resolutivos en circulación tiene varias explicaciones posibles. Una de ellas es que dichos mediadores se concentren en los tejidos dañados y su producción no se refleje en la circulación. Compatibles con esta hipótesis son los reportes de niveles circulantes bajos de LXA4 en pacientes con periodontitis agresiva ${ }^{5}$ y con enfermedad arterial periférica $^{17}$, y a su vez niveles elevados fuera de la circulación, en líquido sinovial de pacientes con $A R,{ }^{18}$ en mucosa intestinal de pacientes con colitis ulcerosa en remisión ${ }^{19}$ y en adipocitos de pacientes obesos, ${ }^{20}$ en donde también se han encontrado RvD1 y RvD2. Opuestos a esta explicación son los reportes en pacientes con Alzheimer, que tienen niveles bajos de LXA4 tanto en circulación como en líquido cefalorraquídeo (LCR), ${ }^{21}$ y en pacientes con esclerosis múltiple, en los que se han detectado niveles elevados de NPD1 tanto en suero como en LCR. ${ }^{22}$ Una segunda explicación de los niveles bajos de RvD1 y de LXA4 en nuestros pacientes con enfermedades isquémicas coronarias es que son incapaces de producir cantidades suficientes de dichos mediadores, lo que contribuiría a una evolución desfavorable de la enfermedad. No se conocen las razones de esta posible falta de producción de mediadores resolutivos. Aún debe dilucidarse si su origen es secundario, por ejemplo, a la propia cronicidad de la inflamación, o se debe a una deficiencia primaria que impediría la resolución de un proceso inflamatorio agudo, favoreciendo su cronicidad, la disfunción y, eventualmente, también las complicaciones agudas del proceso crónico. Compatibles con ello existen reportes de que niveles altos de RvD2 y Mar1 correlacionan con estabilidad de la placa en ratones $A p o e^{-/, 11}$ y por el contrario, en ratones $L d I r^{-1}$ las lesiones avanzadas tienen niveles mayores de RvD1 en comparación con aquellas más tempranas. En este mismo estudio se reporta una correlación negativa entre los niveles de RvD1 y la vulnerabilidad de la placa en carótidas humanas. ${ }^{5}$

De manera opuesta, encontramos concentraciones elevadas de citocinas inflamatorias (IL-1, IL-6 y IL-8) en las dos enfermedades isquémicas agudas, al compararlas con individuos con dolor torácico no cardiogénico, lo que sugiere la presencia de un proceso inflamatorio sistémico en plena actividad. La citocina antiinflamatoria IL-10 también se encontró significativamente elevada en pacientes con IAM-CEST en comparación con el grupo de dolor y con los pacientes con
AE. La correlación positiva altamente significativa entre las concentraciones de IL-10 y las citocinas proinflamatorias sugiere que la intensidad de la respuesta de regulación es directamente proporcional a la intensidad del estímulo inflamatorio, en contraposición al concepto de que, al encontrarse elevada en los SICA, la IL-10 sería un «lobo con ropajes de oveja». ${ }^{23}$ El hallazgo de concentraciones elevadas de MMP en circulación también es compatible con la bien documentada actividad del proceso inflamatorio en los pacientes con enfermedad isquémica coronaria. Ya previamente se ha reportado un vínculo entre la presencia de IL-1 $\beta$, IL-6 e IL-8 con la actividad de MMP, lo que favorecería la degradación de la matriz extracelular que recubre la placa aterosclerosa. ${ }^{24-27}$

En resumen, las dos primeras fases del proceso inflamatorio se encontraron activas, mas no la tercera (resolución). De hecho, todas las citocinas medidas correlacionaron inversamente con RvD1 y con LXA4, aunque solo con esta última alcanzaron significancia estadística. Las MMP también tuvieron una correlación inversa con RvD1 (MMP-2 y MMP-9) y con LXA4 (MMP-9). Ello significa que, en los casos que estudiamos, los mediadores lipídicos de resolución RvD1 y LXA4, lejos de encontrarse aumentados como se esperaría de una respuesta de retorno a la homeostasis tisular ante un grave reto inflamatorio, se encontraron bajos comparados con sujetos con dolor torácico de origen no cardiogénico. Y más llamativo aún es que dichos mediadores se encontraron más bajos en la medida en que era más intensa la respuesta inflamatoria, a juzgar por los niveles de citocinas.

En ese sentido, los mediadores de resolución mostraron pendientes de correlación positiva con la FEVI y negativa con la puntuación GRACE, sin alcanzar significancia estadística. Por el contrario, las pendientes de la correlación de las citocinas y de ET-1 fueron positivas con las puntuaciones GRACE y negativas con la FEVI; ambas estadísticamente significativas. Hay reportes previos en el mismo sentido. Así, los niveles de IL-1 $\beta$ y de IL-8 se han relacionado con disfunción del ventrículo izquierdo, ${ }^{28}$ a la IL-6 se la ha implicado en la patogénesis del SICA por su relación con los niveles de MMP-9 en la placa, ${ }^{24}$ y se han reportado niveles más altos de IL-6 en pacientes con SICA comparados con pacientes con AE. ${ }^{29}$

Una fortaleza de este estudio es que las muestras de sangre se obtuvieron antes del inicio de cualquier tratamiento modificador de la respuesta inflamatoria, lo que evita sesgos por el tratamiento. Las limitaciones del estudio incluyen las diferencias de sexo entre 
los grupos, lo que podría modificar los resultados. Otro factor confusor podría ser el hecho de que en los pacientes con IAM-CEST se encontró mayor proporción de fumadores que en el resto de los grupos. Hasta donde sabemos, debido a que el estudio de los mediadores resolutivos es muy reciente, los kits de ELISA para medirlos no han sido validados contra el método de referencia que es la cromatografía líquida acoplada a espectrometría de masas.

\section{Conclusiones}

En conclusión, la escasa respuesta de resolución en todos los pacientes con SICA estudiados, a pesar de una respuesta inflamatoria intensa, abona en favor de la hipótesis de que estos pacientes tienen una deficiencia que impediría reparar los daños generados por el proceso inflamatorio, y los dejaría vulnerables a la disfunción y a las complicaciones agudas. Es importante probar la veracidad de esta hipótesis, de manera que pudieran plantearse potenciales tratamientos para el manejo de la inflamación cardiovascular crónica.

\section{Financiamiento}

Fondos internos del Instituto Nacional de Cardiología Ignacio Chávez.

\section{Conflictos de intereses}

Ninguno.

\section{Bibliografía}

1. Serhan CN, Brain SD, Buckley CD, Gilroy DW, Haslett C, O'Neill LA, et al. Resolution of inflammation: state of the art, definitions and terms. FASEB J. 2007;21:325-32.

2. Nathan C, Ding A. Nonresolving inflammation. Cell. 2010;140:871-82.

3. Elajami TK, Colas RA, Dalli J, Chiang N, Serhan CN, Welty FK. Specialized proresolving lipid mediators in patients with coronary artery disease and their potential for clot remodeling. FASEB J. 2016;30:2792-801.

4. Sansbury BE, Spite M. Resolution of acute inflammation and the role of resolvins in immunity, thrombosis, and vascular biology. Circ Res. 2016 119:113-30.

5. Fredman G, Hellmann J, Proto JD, Kuriakose G, Colas RA, Dorweiler B, et al. An imbalance between specialized pro-resolving lipid mediators and pro-inflammatory leukotrienes promotes instability of atherosclerotic plaques. Nat Commun. 2016;7:12859.

6. Cannon CP, Brindis RG, Chaitman BR, Cohen DJ, Cross JT Jr, Drozda JP Jr, et al. American College of Cardiology key data elements and definitions for measuring the clinical management and outcomes of patients with acute coronary syndromes: a report of the American College of Cardiology Task
Force on Clinical Data Standards (Acute Coronary Syndromes Writing Committee). J Am Coll Cardiol. 2001;38:2114-30.

7. Libby P, Nahrendorf M, Swirski FK. Leukocytes link local and systemic inflammation in ischemic cardiovascular disease an expanded cardiovascular continuum. J Am Coll Cardiol. 2016;67):1091-103.

8. Libby P. Mechanisms of acute coronary syndromes and their implications for therapy. N Engl J Med. 2013;368:2004-13.

9. Bentzon JF, Otsuka F, Virmani R, Falk E. Mechanisms of plaque formation and rupture. Circ Res. 2014;114:1852-66.

10. Falk E, Nakano M, Bentzon JF, Finn AV, Virmani R. Update on acute coronary syndromes: the pathologists' view. Eur Heart J. 2013;34:719-28.

11. Viola JR, Lemnitzer $P$, Jansen $Y$, Csaba G, Winter $C$, Neideck $C$, et al. Resolving lipid mediators maresin 1 and resolvin D2 prevent atheroprogression in mice. Circ Res. 2016;119:1030-8.

12. Serhan CN. Novel lipid mediators and resolution mechanisms in acute inflammation: to resolve or not? Am J Pathol. 2010;177:1576-91.

13. Chiang N, Fredman G, Bäckhed F, Oh SF, Vickery T, Schmidt BA, et al. Infection regulates pro-resolving mediators that lower antibiotic requirements. Nature. 2012;484:524-8.

14. Wheeler DC, Becker GJ. Summary of KDIGO guideline. What do we really know about management of blood pressure in patients with chronic kidney disease? Kidney Int. 2013;83:377-83.

15. Alnasser SM, Huang W, Gore JM, Steg PG, Eagle KA, Anderson FA Jr, et al. Late consequences of acute coronary syndromes: Global Registry of Acute Coronary Events (GRACE) follow-up. Am J Med. 2015; 128:766-75.

16. Quiñones MA, Otto CM, Stoddard M, Waggoner A, Zoghbi WA. Recommendations for quantification of Doppler echocardiography: a report from the Doppler Quantification Task Force of the Nomenclature and Standards Committee of the American Society of Echocardiography. J Am Soc Echocardiogr. 2002;15:167-84.

17. Ho KJ, Spite M, Owens CD, Lancero H, Kroemer AH, Pande R, et al. Aspirin-triggered lipoxin and resolvin E1 modulate vascular smooth muscle phenotype and correlate with peripheral atherosclerosis. Am J Pathol. 2010;177:2116-23.

18. Giera M, Ioan-Facsinay A, Toes R, Gao F, Dalli J, Deelder AM, et al. Lipid and lipid mediator profiling of human synovial fluid in rheumatoid arthritis patients by means of LC-MS/MS. Biochim Biophys Acta - Mol Cell Biol Lipids. 2012;1821:1415-24.

19. Vong L, Ferraz JG, Dufton N, Panaccione R, Beck PL, Sherman PM, et al. Up-regulation of annexin-A1 and lipoxin A4 in individuals with ulcerative colitis may promote mucosal homeostasis. PLoS One. 2012;7:e39244.

20. Claria J, Dalli J, Yacoubian S, Gao F, Serhan CN. Resolvin D1 and resolvin D2 govern local inflammatory tone in obese fat. J Immunol. 2012; 189:2597-605.

21. Wang X, Zhu M, Hjorth E, Cortés-Toro V, Eyjolfsdottir H, Graff C, et al. Resolution of inflammation is altered in Alzheimer's disease. Alzheimer's Dement. 2015;11:40-50.e2.

22. Prüss, Rosche B, Sullivan AB, Brommer B, Wengert $O$, Gronert K, et al. Proresolution lipid mediators in multiple sclerosis - differential, disease severity-dependent synthesis - a clinical pilot trial. PLoS One 2013;8:e55859.

23. Tziakas DN, Chalikias GK, Kaski JC. Anti-inflammatory cytokines: a wolf in sheep's clothing? Am J Med. 2012;125:e19

24. Shu J, Ren N, Du J-B, Zhang M, Cong H-L, Huang T-G. Increased levels of interleukin- 6 and matrix metalloproteinase- 9 are of cardiac origin in acute coronary syndrome. Scand Cardiovasc J. 2007;41:149-54.

25. Gough PJ, Gomez IG, Wille PT, Raines EW. Macrophage expression of active MMP-9 induces acute plaque disruption in apoE-deficient mice. J Clin Invest. 2006;116:59-69.

26. Mountain DJH, Singh M, Menon B, Singh K. Interleukin-1 beta increases expression and activity of matrix metalloproteinase-2 in cardiac microvascular endothelial cells: role of PKCalpha/beta1 and MAPKs. Am J Physiol Cell Physiol. 2007;292:C867-75

27. Derosa G, D'Angelo A, Scalise F, Avanzini MA, Tinelli C, Peros E, et al. Comparison between metalloproteinases-2 and -9 in healthy subjects, diabetics, and subjects with acute coronary syndrome. Heart Vessels. 2007;22:361-70.

28. Zarrouk-Mahjoub S, Zaghdoudi M, Amira Z, Chebi H, Khabouchi N, Finsterer $\mathrm{J}$, et al. Pro- and anti-inflammatory cytokines in post-infarction left ventricular remodeling. Int J Cardiol. 2016;221:632-6.

29. Heinisch RH, Zanetti CR, Comin F, Fernandes JL, Ramires JA, Serrano CV. Serial changes in plasma levels of cytokines in patients with coronary artery disease. Vasc Health Risk Manag. 2005;1:245-50. 